\title{
MicroRNA-24-3p inhibition prevents cell growth of vascular smooth muscle cells by targeting Bcl-2-like protein 11
}

\author{
HUANXIN ZHANG* ${ }^{*}$ SHIZHEN XUE* , YI FENG, JUN SHEN and JIXIAN ZHAO \\ Department of Cardiology, Renmin Hospital, Hubei University of Medicine, Shiyan, Hubei 442000, P.R. China
}

Received April 24, 2019; Accepted November 29, 2019

DOI: $10.3892 /$ etm.2020.8517

\begin{abstract}
Numerous reports have shown that dysfunction of vascular smooth muscle cells (VSMCs) serves a critical function in the development of cardiovascular disease, including coronary heart disease (CHD). microRNAs (miRNAs/miRs) have been reported to play important roles in regulating the function of VSMCs. The present study aimed to determine the role of miR-24-3p in VSMCs and to uncover the underlying mechanism. The expression of miR-24-3p in the peripheral blood samples of CHD patients was measured by reverse transcription-quantitative (RT-q) PCR. It was found that the level of miR-24-3p in the peripheral blood of patients with CHD was significantly upregulated compared with that in healthy controls. A dual luciferase reporter assay was performed to determine whether Bcl-2-like protein 11 (Bcl-2L11) was a target gene of miR-24-3p, and it was identified that Bcl-2L11 was a direct target of miR-24-3p. The mRNA level and protein expression of Bcl-2L11 in the peripheral blood of patients with CHD were measured by RT-qPCR and western blotting, respectively. The findings suggested that Bcl-2L11 was downregulated in the peripheral blood of patients with CHD. In addition, it was found that downregulation of miR-24-3p suppressed VSMC proliferation and promoted VSMC apoptosis, while the effects of the miR-24-3p inhibitor on cell viability and apoptosis were reversed by Bcl-2L11-small interfering (si)RNA. Additionally, downregulation of miR-24-3p increased the levels of Bcl-2L11, caspase-3 and Bax, and decreased Bcl-2 expression in VSMCs; these changes were abolished by Bcl-2L11-siRNA. In conclusion, the aforementioned results indicated that miR-24-3p was an important regulator in VSMC prolifera-
\end{abstract}

Correspondence to: Dr Yi Feng, Department of Cardiology, Renmin Hospital, Hubei University of Medicine, 39 Chaoyang Middle Road, Shiyan, Hubei 442000, P.R. China

E-mail: fengyi773319@163.com

* Contributed equally

Key words: microRNA-24-3p, vascular smooth muscle cells, coronary heart disease, Bcl-2L11 tion and apoptosis by targeting Bcl-2L11, which suggested that miR-24-3p might be a potential therapeutic target for the treatment of CHD.

\section{Introduction}

Cardio- and cerebrovascular disease incidence has increased due to improved living standards $(1,2)$. Coronary heart disease (CHD) is one of the leading causes of death in the world and adversely affects public health $(3,4)$. An increasing number of studies have indicated that vascular smooth muscle cells (VSMCs) serve vital functions in the development of CHD $(5,6)$. However, the molecular mechanisms of VSMCs in CHD have yet to be elucidated.

microRNAs (miRNAs/miRs) are a class of non-coding RNAs of 20-22 nucleotides in length that are able to regulate $30-50 \%$ of all genes by binding to the 3 '-untranslated regions (3'-UTRs) (7). miRNAs regulate various biological functions, including cell proliferation, apoptosis and signal transduction (8). Previous studies have suggested that miRNAs participate in the pathological processes of a number of diseases, including cardiac hypertrophy (9), heart failure (10), myocardial ischemia (11) and reperfusion (12). Previous studies have demonstrated that miR-24 is a vital molecule in mediating vascular endothelial cells $(13,14)$. miR-24-3p has been shown to play an important role in regulating cell growth and metastasis in various types of cancer (15-17). In addition, miR-24-3p is involved in ischemia/reperfusion injury in cardiomyocytes (18). However, the function of miR-24-3p in VSMCs in coronary heart disease remains to be elucidated.

The Bcl-2 and caspase protein families serve different and vital roles in cell apoptosis (19). Bcl-2-like protein 11 (Bcl-2L11; $\mathrm{Bim})$ is a pro-apoptotic member of the Bcl-2 family, which induces cytochrome c release from the mitochondria (20). Previous studies have reported that Bcl-2L11 mediates the biological processes of cell growth and apoptosis (21-23). Therefore, the present study investigated the modulating effect of miR-24-3p in VSMCs. Furthermore, the underlying mechanism by which miR-24-3p regulated the apoptosis of VSMCs was clarified.

The purpose of the present study was to investigate the expression of miR-24-3p in the blood samples of CHD patients, examine the role of miR-24-3p in VSMCs, and further to explore the molecular mechanism. 


\section{Materials and methods}

Clinical specimen collection. Blood samples were collected from 30 patients with coronary heart disease ( 22 male, 8 female; age range, $37-75$ years) and 30 healthy volunteers (22 male, 8 female; age range: $34-73$ years) from Renmin Hospital (Shiyan, China) between February 2016 and June 2018. The specimens were rapidly frozen and stored at $-80^{\circ} \mathrm{C}$ until use. All patients provided written informed consent and approved the use of their samples in the present study. The study procedures obtained approval from the Ethics Committee at Renmin Hospital.

Cell culture. Human VSMCs were obtained from the American Type Culture Collection (cat. no. ATCC ${ }^{\circledR}$ PCS-100-012). Cells were cultured in DMEM (Gibco; Thermo Fisher Scientific, Inc.) containing 10\% FBS (Gibco; Thermo Fisher Scientific, Inc.) and $1 \%$ penicillin/streptomycin (Sigma-Aldrich; Merck $\mathrm{KGaA})$ at $37^{\circ} \mathrm{C}$ in a $5 \% \mathrm{CO}_{2}$ incubator.

Cell transfection and reagents. In total, $100 \mathrm{nM}$ miR-24-3p inhibitor (antagonist of miR-24-3p; 5'-CUGUUCCUGCUG AACUGAGCCA-3'), $100 \mathrm{nM}$ inhibitor control (5'-CAGUAC UUUUGUGUAGUACAA-3'), $100 \mathrm{nM}$ miR-24-3p mimic (sense: 5'-UGGCUCAGUUCAGCAGGAACAG-3'; anti-sense: 5'-GUU CCUGCUGAACUGAGCCAUU-3), $100 \mathrm{nM}$ mimic control (sense: 5'-UUCUCCGAACGUGUCACGUTT-3'; anti-sense: 5'-ACGUGACACGUUCGGAGAATT-3'; all from Shanghai GenePharma Co., Ltd.), $1 \mu \mathrm{M}$ control small interfering (si)RNA (cat. no. sc-36869; Santa Cruz Biotechnology, Inc.), $0.2 \mu \mathrm{M}$ Bcl-2L11-siRNA (cat. no. sc-29802; Santa Cruz Biotechnology, Inc.), $100 \mathrm{nM}$ miR-24-3p inhibitor $+1 \mu \mathrm{M}$ control-siRNA, or $100 \mathrm{nM}$ miR-24-3p inhibitor $+0.2 \mu \mathrm{M}$ Bcl-2L11-siRNA were transfected into VSMCs using Lipofectamine ${ }^{\circledR} 2000$ (Invitrogen; Thermo Fisher Scientific, Inc.) for in accordance with the manufacturer's protocol. Reverse transcription-quantitative (RT-q) PCR was performed to detect the efficiency of cell transfection $48 \mathrm{~h}$ after incubation, at $37^{\circ} \mathrm{C}$.

CCK-8 assay. A Cell Counting Kit-8 (Beyotime Institute of Biotechnology) was employed to detect the cell viability of VSMCs, according to the manufacturer's protocol. Cells were seeded into 96 -well culture plates $\left(6 \times 10^{3}\right.$ cells/well) and then incubated in DMEM for $24 \mathrm{~h}$ at $37^{\circ} \mathrm{C}$. Then, the cells were transfected with inhibitor control, miR-24-3p inhibitor, miR-24-3p inhibitor + control-siRNA, or miR-24-3p inhibitor + Bcl-2L11-siRNA for $48 \mathrm{~h}$. Subsequently, CCK-8 reagent was added into each well and the cells were incubated at $37^{\circ} \mathrm{C}$ for another $2 \mathrm{~h}$. The absorbance (optical density) at a wavelength of $450 \mathrm{~nm}$ was measured using a microplate reader (Eon; BioTek Instruments, Inc.).

Flow cytometry analysis. VSMCs were transfected with inhibitor control, miR-24-3p inhibitor, miR-24-3p inhibitor + control-siRNA, or miR-24-3p inhibitor + Bcl-2L11-siRNA for $48 \mathrm{~h}$. Then, Annexin V-FITC/propidium iodide (PI) dual staining was performed to evaluate cell apoptosis, according to the manufacturer's protocol (cat. no. KGA106; Nanjing KeyGen Biotech Co., Ltd.). Briefly, VSMCs were digested with $0.2 \%$ trypsin, washed with PBS and fixed with $70 \%$ ethanol overnight at $4^{\circ} \mathrm{C}$. Then, the cells were stained with $5 \mu \mathrm{l}$
Annexin V-FITC and $5 \mu \mathrm{l}$ PI for $30 \mathrm{~min}$ at room temperature. Finally, the stained cells were quantified using a FACSCalibur flow cytometer (BD Biosciences) and the data were analyzed with FlowJo 7.6.1 software (FlowJo LLC).

Dual-luciferase reporter assay. A bioinformatics prediction program (TargetScan 7.2; http://www.targetscan.org/vert_72/) was used to predict the relationship between miR-24-3p and Bcl-2L11, and binding sites between miR-24-3p and Bcl-2L11 were observed. To confirm the prediction, the wild-type (WT-Bcl-2L11: 5'-CCCCUGCAGUGGAAACUGAGCCA-3') and mutant (MUT-Bcl-2L11: 5'-CCAAGCAAGUGGAAAAGCGCAAG-3') 3'UTR of Bcl-2L11, containing the miR-24-3p-binding elements, were generated by RT-PCR using a Transcriptor First Strand cDNA Synthesis Kit (Roche Molecular Systems, Inc.) from total RNA preps extracted from VSMCs, using the temperature protocol of $5 \mathrm{~min}$ at $25^{\circ} \mathrm{C}$ followed by $60 \mathrm{~min}$ at $42^{\circ} \mathrm{C}$. The sequences were then cloned into a pmiR-RB-Report ${ }^{\mathrm{TM}}$ dual luciferase reporter gene plasmid vector (Guangzhou RiboBio Co., Ltd.). Then $100 \mathrm{ng}$ Bcl-2L11-WT or $100 \mathrm{ng} \mathrm{Bcl-2L11-MUT} \mathrm{were} \mathrm{co-transfected} \mathrm{with}$ $100 \mathrm{nM}$ miR-24-3p mimic or $100 \mathrm{nM}$ mimic control into VSMCs using Lipofectamine ${ }^{\circledR} 2000$ (Invitrogen; Thermo Fisher Scientific, Inc.), according to the manufacturer's protocols. Dual-Luciferase ${ }^{\circledR}$ Reporter Assay kit (Promega Corporation) was used to measure luciferase activity $48 \mathrm{~h}$ after cell transfection, which were normalized to that of Renilla luciferase.

$R T$-qPCR. TRIzol ${ }^{\circledR}$ reagent (Invitrogen; Thermo Fisher Scientific, Inc.) was used to isolate the total RNA from cells or blood samples, respectively, according to the manufacturer's protocol. Then, $200 \mathrm{ng}$ total RNA was reverse transcribed into cDNA using the miScript RT kit (Applied Biosystems; Thermo Fisher Scientific, Inc.), following the manufacturer's protocol. The temperature protocol for the reverse transcription reaction was as follows: Initial annealing at $25^{\circ} \mathrm{C}$ for $5 \mathrm{~min}$, followed by extension at $42^{\circ} \mathrm{C}$ for $60 \mathrm{~min}$ and termination at $80^{\circ} \mathrm{C}$ for $2 \mathrm{~min}$. The expression levels of miR-24-3p and Bcl-2L11 were quantified using a SYBR Green PCR Master Mix kit (Takara Biotechnology Co., Ltd.). GAPDH and U6 were used to normalize mRNA and miR-24-3p expression, respectively. The reaction conditions of the qPCR were as follows: $95^{\circ} \mathrm{C}$ for $5 \mathrm{~min} ; 35$ cycles of denaturation at $94^{\circ} \mathrm{C}(15 \mathrm{sec})$, annealing at $50^{\circ} \mathrm{C}$ for $30 \mathrm{sec}$ and chain extension at $72^{\circ} \mathrm{C}$ for $30 \mathrm{sec}$; and a final extension step at $72^{\circ} \mathrm{C}$ for $10 \mathrm{~min}$. Primers were purchased from Sangon Biotech Co., Ltd. (Shanghai, China): U6 forward, 5'-GCTTCGGCAGCA CATATACTAAAAT-3' and reverse, 5'-CGCTTCACGAAT TTGCGTGTCAT-3'; GAPDH forward, 5'-TGTTGCCATCAA TGACCCCTT-3' and reverse, 5'-CTCCACGACGTACTCAG CG-3'; miR-24-3p forward, 5'-ACACTCCAGCTGGGTGGC TCAGTTCAGCAG-3' and reverse, 5'-CTCAACTGGTGTCGT GGAGTCGGCAATTCAG-3'; Bcl-2L11 forward, 5'-CACAAA CCCCAAGTCCTCCT-3' and reverse, 5'-ACACCAGGCGGA CAATGTAA-3'; caspase-3 forward, 5'-TGTCGATGCAGC AAACCTCA-3' and reverse, 5'-GACTTCTACAACGATCCC CTC-3'; Bax forward, 5'-CGTCCACCAAGAAGCTGAGCG-3' and reverse, 5'-CGTCCACCAAAGCTGAGCG-3'; and Bcl-2 forward, 5'-TTGGATCAGGGAGTTGGAAG-3' and reverse,

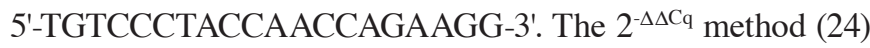
was used to calculate the relative expression levels. The assay was repeated three times. 
Western blot analysis. Proteins from VSMCs or blood samples were extracted using RIPA buffer (Beyotime Institute of Biotechnology). The protein concentration was detected by BCA Protein Assay kit (Thermo Fisher Scientific, Inc.). Then, the extracted protein samples were mixed with $5 \mathrm{X}$ loading buffer, boiled at $100^{\circ} \mathrm{C}$ for $5 \mathrm{~min}$, centrifuged at $1,000 \mathrm{x} \mathrm{g}$ at $4^{\circ} \mathrm{C}$ for $2 \mathrm{~min}$. Protein samples (30 $\mu \mathrm{g}$ protein/lane) were separated by $10 \%$ SDS-PAGE and subsequently transferred onto a PVDF membrane. Then, the membranes were blocked with $5 \%$ skimmed milk at room temperature for $1.5 \mathrm{~h}$ and subsequently incubated with the primary antibodies at $4^{\circ} \mathrm{C}$ overnight: Bcl-2L11 (cat. no. 2933; dilution 1:1,000), caspase-3 (cat. no. 14220; dilution 1:1,000), Bcl-2 (cat. no. 4223; dilution 1:1,000), Bax (cat. no. 5023; dilution 1:1,000) and GAPDH (cat. no. 5174; dilution 1:1,000; all from Cell Signaling Technology Inc.). After washing with TBS with Tween-20, the membranes were incubated with horseradish peroxidase-conjugated anti-rabbit immunoglobulin G secondary antibody (cat. no. 7074; dilution: 1:2,000; Cell Signaling Technology, Inc.) for $2 \mathrm{~h}$ at room temperature. Finally, the protein bands were visualized using ECL reagent (EMD Millipore), according to the manufacturer's protocols.

Statistical analysis. All the aforementioned experiments were performed in triplicate. Data are expressed as the mean \pm SD. Statistical analysis was performed using SPSS 19.0 software (IBM Corp.). Comparisons between groups were estimated by Student's t-test or one-way analysis of variance followed by Tukey's post hoc test. $\mathrm{P}<0.05$ was considered to indicate a statistically significant difference.

\section{Results}

miR-24-3p expression is upregulated in blood samples from patients with $C H D$. The present study first evaluated miR-24-3p expression levels in peripheral blood samples from 30 patients with CHD and normal controls. The results from RT-qPCR indicated that the miR-24-3p expression was significantly higher in the peripheral blood samples of patients with CHD than that in the healthy volunteers (Fig. 1).

Bcl-2L11 is a direct target of $m i R-24-3 p$. To investigate potential miR-24-3p target sites, TargetScan was used to analyze the target genes of miR-24-3p. It was found that Bcl-2L11 was a possible target of miR-24-3p and the binding sites are shown in Fig. 2A. To better understand the interaction between miR-24-3p and Bcl-2L11, a luciferase reporter gene assay was performed. The results suggested that miR-24-3p mimic markedly suppressed the luciferase activity of cells co-transfected with miR-24-3p mimic and Bcl-2L11-WT, whereas no significant differences were observed in luciferase activity in cells co-transfected with miR-24-3p mimic and Bcl-2L11-MUT (Fig. 2B).

Bcl-2L11 mRNA and protein levels were then detected in the blood samples of patients with CHD using RT-qPCR and western blotting. As shown in Fig. 2C, the mRNA level of Bcl-2L11 was significantly reduced in the blood samples of patients with CHD compared with healthy controls. In addition, a relatively decreased protein expression level of Bcl-2L11 was observed in the blood samples of CHD patients via western

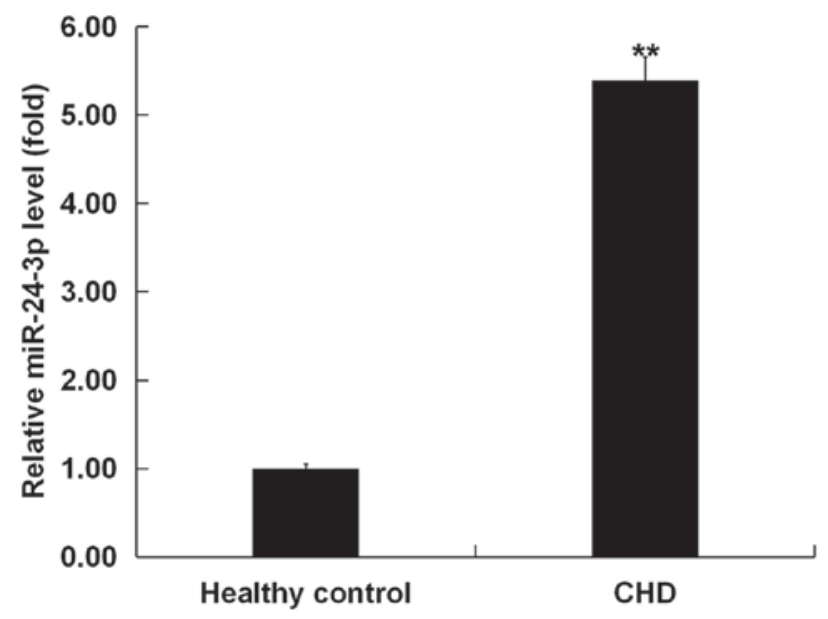

Figure 1. miR-24-3p is upregulated in the blood samples of patients with CHD. The expression level of miR-24-3p was determined by reverse transcription-quantitative PCR in the peripheral blood samples from 30 patients with CHD and 30 healthy controls. All experiments were performed three times. Data are presented as the mean $\pm \mathrm{SD} .{ }^{* *} \mathrm{P}<0.01$ vs. healthy control miR, microRNA; CHD, coronary heart disease.

blot analysis (Fig. 2D). In summary, it was concluded that Bcl-2L11 was a direct target of miR-24-3p. The expression levels of Bcl-2L11 in patients with CHD were reduced.

Bcl-2L11-siRNA reverses the incremental effects of $m i R-24-3 p$ inhibitor on Bcl-2L11 expression in VSMCs. The functional relevance of Bcl-2L11 in miR-24-3p-regulated effects in VSMCs was explored. Control-siRNA, Bcl-2L11-siRNA, inhibitor control, miR-24-3p inhibitor, miR-24-3p inhibitor + control-siRNA, or miR-24-3p inhibitor+Bcl-2L11-siRNA were transfected into VSMCs for $48 \mathrm{~h}$. RT-qPCR was performed to evaluate the transfection efficiency. As presented in Fig. 3A, compared to the control group, the levels of miR-24-3p were significantly decreased in VSMCs transfected with miR-24-3p inhibitor. The Bcl-2L11 mRNA level was significantly decreased in VSMCs transfected with Bcl-2L11-siRNA compared with the control group (Fig. 3B). The results of the RT-qPCR and western blotting demonstrated that the mRNA and protein levels of Bcl-2L11 increased in the VSMCs transfected with miR-24-3p inhibitor compared with the control group, and this increase was reversed by Bcl-2L11-siRNA (Fig. 3C and D). Taken together, it was found that Bcl-2L11 was negatively regulated by miR-24-3p in VSMCs.

Bcl-2L11-siRNA reverses the effect of miR-24-3p inhibitor on cell proliferation and apoptosis in VSMCs. In order to further investigate the effect of miR-24-3p on VSMCs, a CCK- 8 assay and flow cytometry were performed to assess VSMC viability and apoptosis. VSMCs were transfected with an inhibitor control, miR-24-3p inhibitor, miR-24-3p inhibitor + control-siRNA, or miR-24-3p inhibitor + Bcl-2L11-siRNA for $48 \mathrm{~h}$. The results of the CCK- 8 analysis indicated that cell viability was significantly decreased in the miR-24-3p inhibitor group compared with the control group, whereas this decrease was reversed by Bcl-2L11-siRNA (Fig. 4A). Flow cytometry analysis demonstrated that compared with the control group, miR-24-3p inhibitor significantly induced 
A

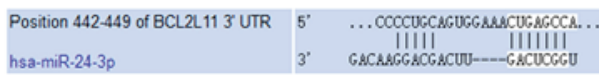

B
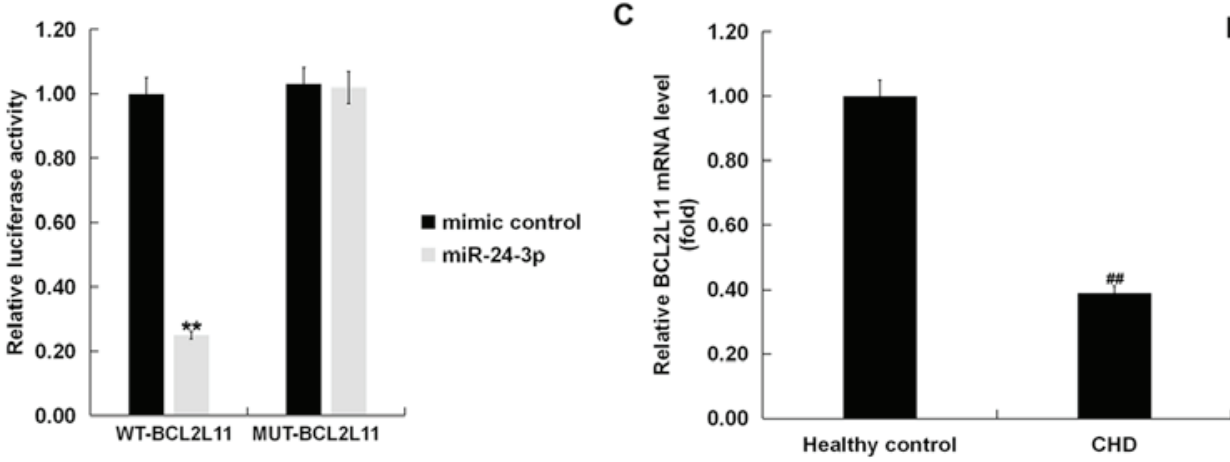

D

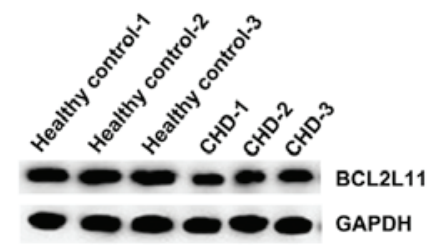

Figure 2. miR-24-3p targets the Bcl-2L11 transcript 3'UTR. (A) Putative target sites of miR-24-3 in the 3'-UTR of Bcl-2L11 predicted by TargetScan. (B) Relative luciferase activities were detected by dual luciferase assay. (C) Reverse transcription-quantitative PCR was performed to detect the mRNA level of Bcl-2L11 in peripheral blood samples of 30 patients with CHD and 30 healthy controls. (D) The protein expression of Bcl-2L11 in the peripheral blood samples of 3 patients with CHD and 3 healthy controls was determined by western blot analysis. All experiments were performed three times. The results are presented as the mean $\pm \mathrm{SD} .{ }^{* *} \mathrm{P}<0.01$ vs. mimic control; ${ }^{\# \#} \mathrm{P}<0.01$ vs. healthy control. miR, microRNA; Bcl-2L11, Bcl-2-like protein 11; UTR, untranslated region; CHD, coronary heart disease.

A

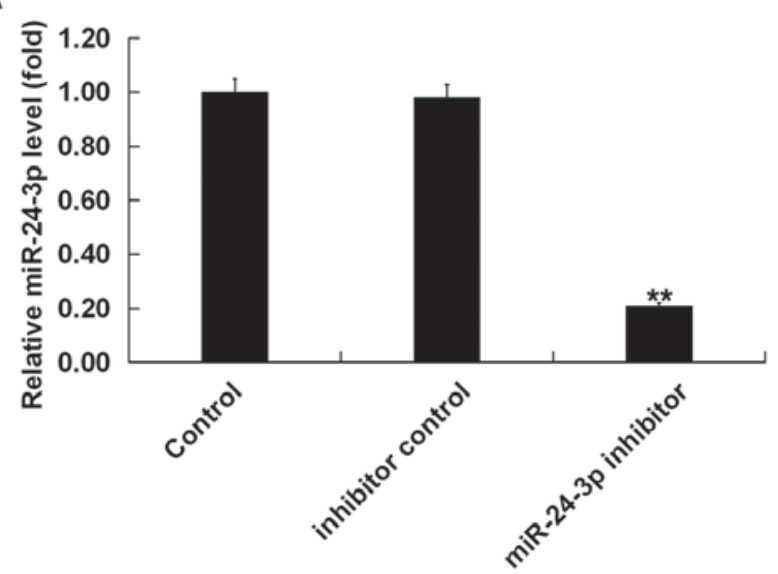

C

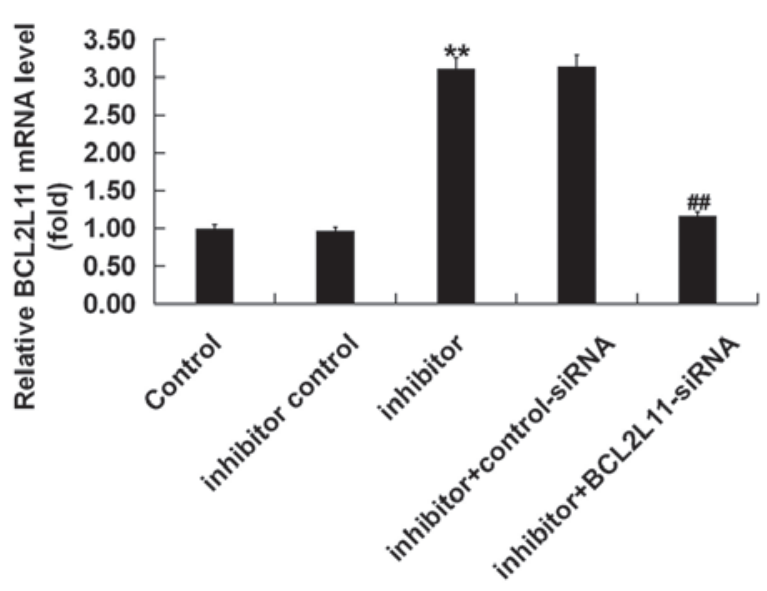

B

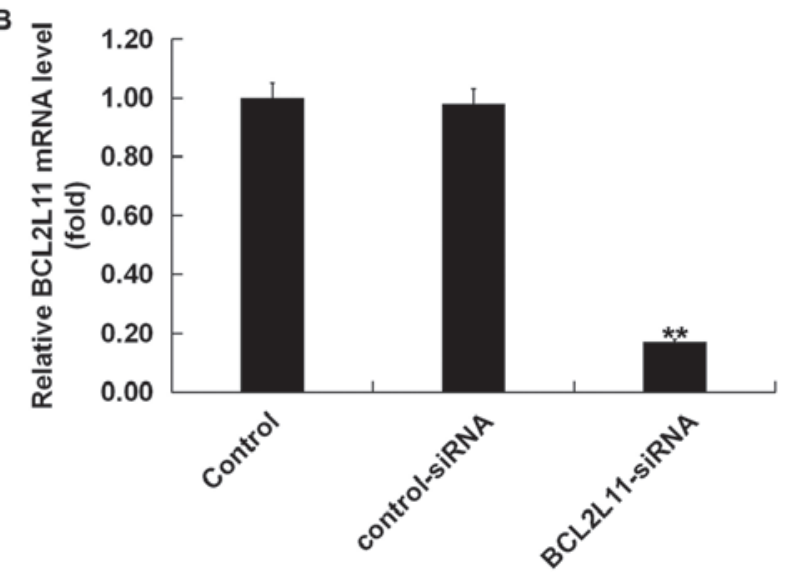

D

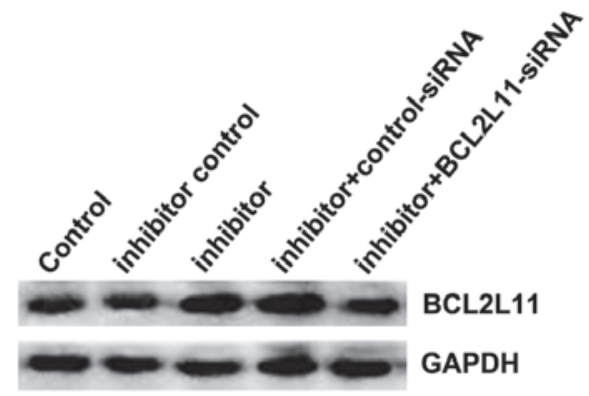

Figure 3. Effect of miR-24-3p inhibitor on Bcl-2L11 expression in VSMCs. VSMCs were co-transfected with the control-siRNA, Bcl-2L11-siRNA, inhibitor control or miR-24-3p inhibitor, miR-24-3p inhibitor + control-siRNA, or miR-24-3p inhibitor + Bcl-2L11-siRNA for $48 \mathrm{~h}$. RT-qPCR was performed to evaluate the transfection efficiency. (A) The level of miR-24-3p was detected when VSMCs were transfected with the miR-24-3p inhibitor or inhibitor control for 48 $\mathrm{h}$. (B) The Bcl-2L11 mRNA level was tested by RT-qPCR in VSMCs transfected with Bcl-2L11-siRNA or control-siRNA. (C) RT-qPCR and (D) western blotting were performed to measure the Bcl-2L11 mRNA and protein expression in different groups. All experiments were performed three times. Data are presented as the mean $\pm \mathrm{SD} .{ }^{* *} \mathrm{P}<0.01$ vs. control. ${ }^{\# \#} \mathrm{P}<0.01$ vs. inhibitor. miR, microRNA; Bcl-2L11, Bcl-2-like protein 11; VSMCs, vascular smooth muscle cells; si, small interfering; RT-qPCR, reverse transcription quantitative PCR. 
A

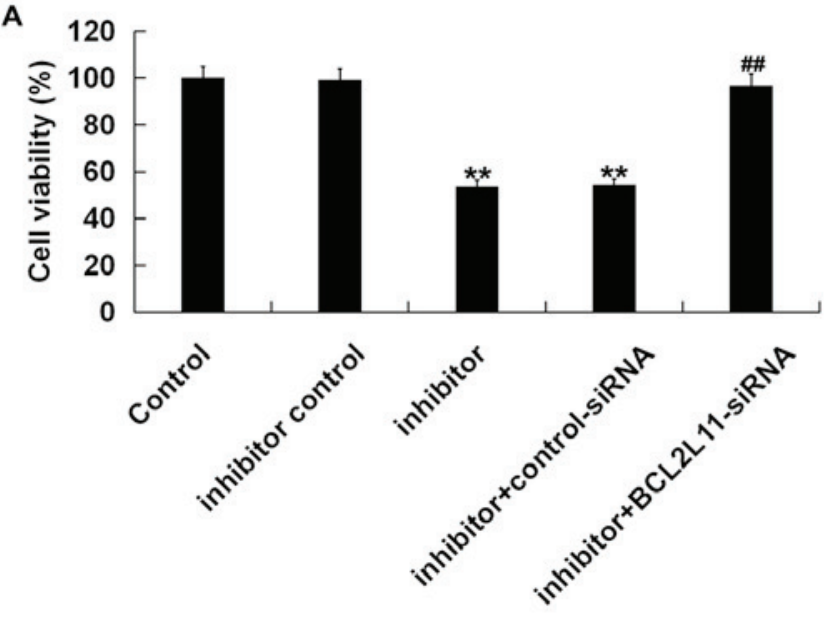

B

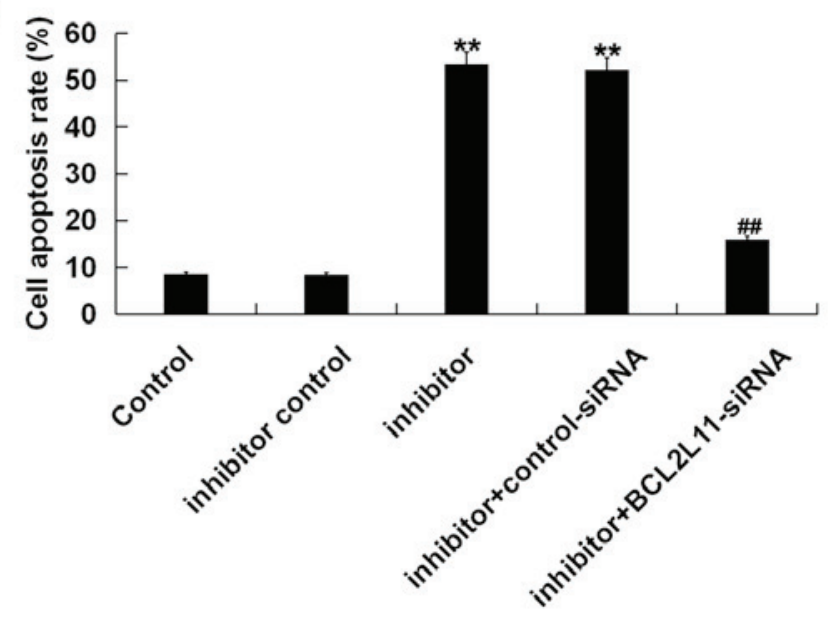

C

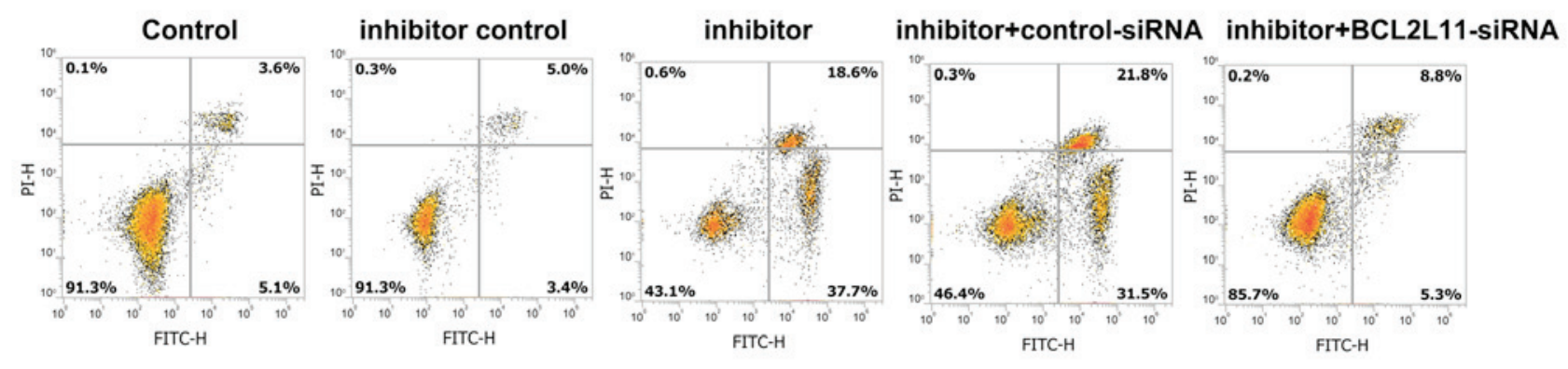

Figure 4. Effects of miR-24-3p inhibitor or Bcl-2L11-siRNA on the cell viability and apoptosis of VSMCs. VSMCs were transfected with the inhibitor control, miR-24-3p inhibitor, miR-24-3p inhibitor + control-siRNA or miR-24-3p inhibitor + Bcl-2L11-siRNA for 48 h. (A) A CCK-8 assay was used to determine the cell viability of VSMCs; (B) the percentages of apoptosis in VSMCs were measured by (C) flow cytometry. All experiments were performed in triplicate. The results are shown as the mean $\pm \mathrm{SD} .{ }^{* *} \mathrm{P}<0.01$ vs. control. ${ }^{\# \#} \mathrm{P}<0.01$ vs. inhibitor. miR, microRNA; Bcl-2L11, Bcl-2-like protein 11 ; VSMCs, vascular smooth muscle cells; si, small interfering; PI, propidium iodide.

apoptosis in VSMCs, while Bcl-2L11-siRNA clearly reversed these effects (Fig. 4B and C). These data demonstrated that miR-24-3p inhibitor could inhibit VSMC viability and induce VSMC apoptosis by targeting Bcl-2L11.

miR-24-3p affects VSMC apoptosis by regulating Bcl-2L11/Bcl-2/Bax/caspase-3 expression. To further explore the underlying mechanism of miR-24-3p inhibitor-induced cell apoptosis, the expression levels of Bcl-2L11, caspase-3, Bcl-2 and Bax were determined by western blot analysis and RT-qPCR. VSMCs were transfected with inhibitor control, miR-24-3p inhibitor, miR-24-3p inhibitor + control-siRNA, or miR-24-3p inhibitor + Bcl-2L11-siRNA for $48 \mathrm{~h}$. As shown in Fig. 5A, miR-24-3p inhibitor increased the protein levels of Bcl-2L11, caspase-3 and Bax, while it decreased the protein levels of Bcl-2 in VSMCs, and these changes were reversed by Bcl-2L11-siRNA. RT-qPCR assay indicated that miR-24-3p inhibitor significantly increased the mRNA levels of Bcl-2L11, caspase- 3 and Bax, while it decreased the mRNA levels of Bcl-2 (Fig. 5). All these changes were reversed by Bcl-2L11-siRNA.

\section{Discussion}

VSMCs are the main cell type in blood vessels and, abnormal proliferation and apoptosis of VSMCs may result in the rapid development of disease $(25,26)$. Recently, growing evidence has identified miRNAs as new biomarkers for a number of cardiovascular diseases $(27,28)$. It is necessary to identify disease-specific miRNAs and their targets to understand their roles in disease (29-31). It has been reported that various miRNAs participate in mediating the functions of VSMCs, including miR-21, miR-214 and miR-146a (32-34). In the present study, the expression level of miR-24-3p in the blood samples of 30 patients with CHD was explored using RT-qPCR and it was found that the miRNA-24-3p level was higher in the blood samples of patients with CHD compared with the normal controls. The results demonstrated that miRNA-24-3p might play a vital role in modulating CHD. Luciferase reporter analysis identified that Bcl-2L11 was a direct target of miRNA-24-3p in VSMCs. The measurements of the mRNA and protein Bcl-2L11 expression in peripheral blood samples of CHD patients and normal volunteers showed that Bcl-2L11 was downregulated in the blood samples of CHD patients. However, the relationship between miRNA-24-3p expression and Bcl-2L11 expression in patients with CHD was not analyzed. This might be a limitation of the present study, and is something to explore in the future.

Then, the role and mechanism of miRNA-24-3p in regulating VSMCs was investigated. It has been reported that Bcl-2L11 is a pro-apoptotic Bcl-2 family member and it is activated in a number of activities, including mediating excitotoxic apoptosis, mitochondrial depolarization and factor 
A

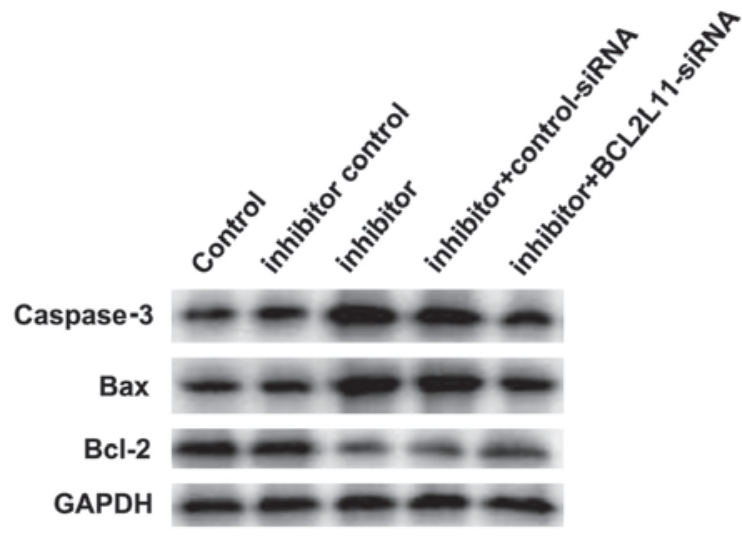

C

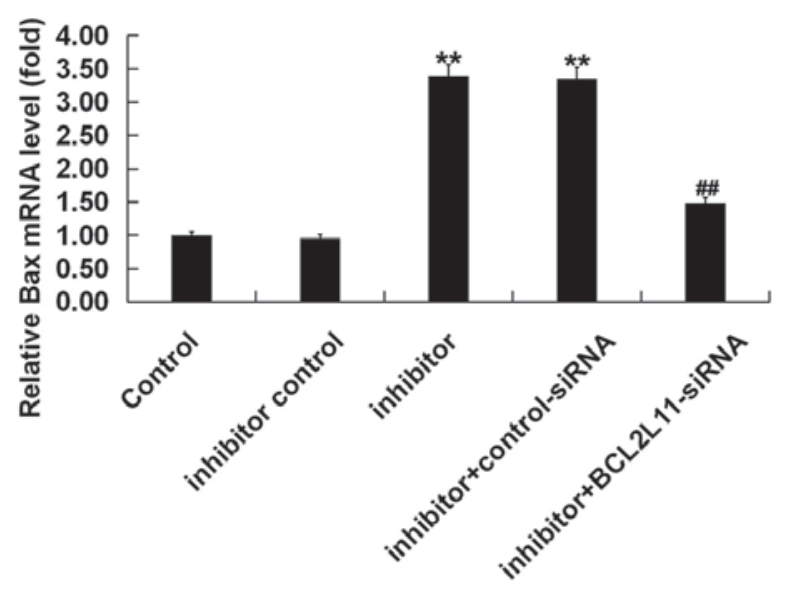

B

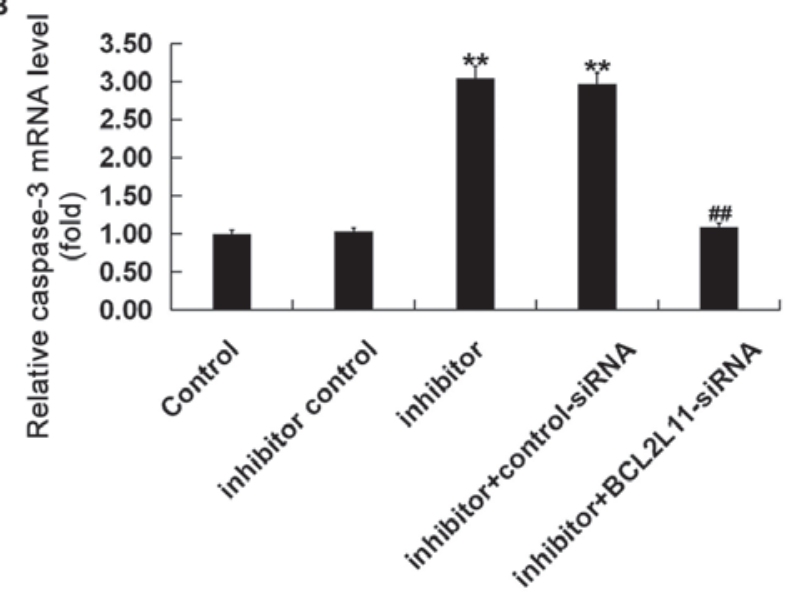

D

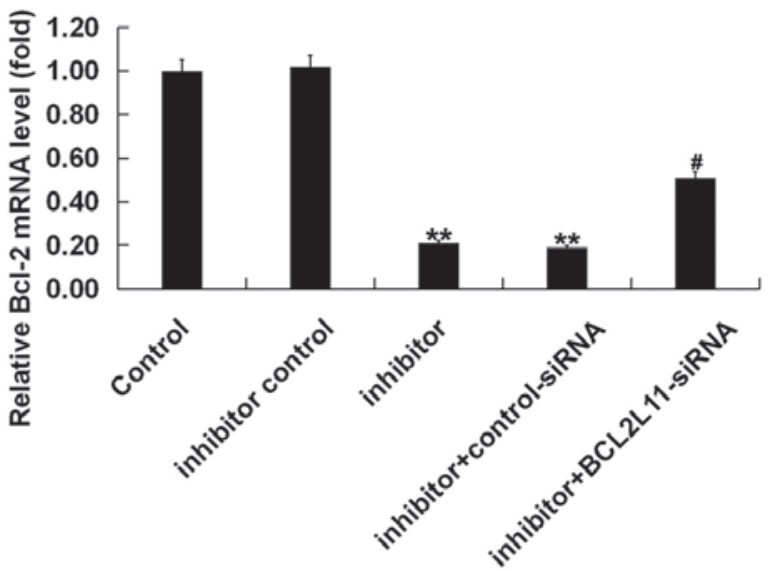

Figure 5. Expression of Bcl-2L11/Bcl-2/Bax/caspase-3 in response to miR-24-3p inhibitor or Bcl-2L11-siRNA transfection in VSMCs. VSMCs were transfected with the inhibitor control, miR-24-3p inhibitor, miR-24-3p inhibitor + control-siRNA or miR-24-3p inhibitor + Bcl-2L11-siRNA for 48 h. (A) The protein levels of caspase-3, Bax, and Bcl-2 were detected by western blotting. The mRNA expression level of (B) caspase-3, (C) Bax and (D) Bcl-2 was detected by reverse transcription-quantitative PCR. All experiments were performed in triplicate. ${ }^{* * *} \mathrm{P}<0.01$ vs. control. ${ }^{\#} \mathrm{P}<0.05$ and ${ }^{\# \#} \mathrm{P}<0.01$ vs. inhibitor. Bcl-2L11, Bcl-2-like protein 11; miR, microRNA; si, small interfering; VSMCs, vascular smooth muscle cells.

translocation (35-37). As Bcl-2L11 was found to be a direct target of miRNA-24-3p in VSMCs, it was hypothesized that altering the expression of miRNA-24-3p in the VSMCs of CHD patients could change the Bcl-2L11 expression and the growth of VSMCs. To test this hypothesis, inhibitor control, miRNA-24-3p inhibitor, miRNA-24-3p inhibitor + control-siRNA, or miRNA-24-3p inhibitor + Bcl-2L11-siRNA were transfected into VSMCs. The results showed that miRNA-24-3p inhibitor significantly increased Bcl-2L11 expression in VSMCs, while this increase was eliminated by Bcl-2L11-siRNA. miRNA-24-3p inhibitor significantly suppressed cell viability and induced apoptosis in VSMCs. However, Bcl-2L11-siRNA significantly reversed the effects of miRNA-24-3p inhibitor on cell viability and cell apoptosis in VSMCs. These data indicated that miRNA-24-3p regulated the apoptosis of VSMCs in patients with CHD. This was in accordance with the observations of previous studies $(38,39)$. However, the effect of miR-24-3p upregulation on VSMCs was not investigated and this might be a limitation of the present study, to be addressed in the future.

Cell apoptosis occurs through two pathways: The death receptor-regulated external signaling pathway and the mitochondria-regulated internal signaling pathway (40). The present study investigated the signaling pathway in which miRNA-24-3p regulated apoptosis in the VSMCs of patients with CHD. It was observed that miRNA-24-3p inhibitor significantly upregulated the expression of Bcl-2L11, caspase-3 and Bax in VSMCs, and the expression of Bcl-2 was suppressed. The effects of miR-24-3p inhibitor on the expression of these genes were reversed by Bcl-2L11-siRNA.

In conclusion, the present study suggested that miRNA-24-3p exhibited a vital role in regulating the viability and apoptosis of VSMCs by targeting Bcl-2L11. This may provide potential therapeutic targets for the interference and treatment of CHD. However, the current study is only a preliminary study of the expression of miRNA-24-3p in CHD patients and its role in VSMCs. To substantiate the role of miRNA-24-3p in CHD, more detailed research is needed. For example, other targets of miR-24-3p in CHD should be investigated to fully demonstrate the function of miRNA-24-3p in VSMCs. The effect of Bcl-2L11-siRNA and miR-24-3p mimic on VSMCs should be investigated. In addition, the role of miRNA-24-3p in CHD in vivo needs further study. 


\section{Acknowledgements}

Not applicable.

\section{Funding}

No funding was received.

\section{Availability of data and materials}

All data sets used and/or generated during the current study are available from the corresponding author on reasonable request.

\section{Authors' contributions}

HXZ and SZX contributed to study design, data collection, statistical analysis, data interpretation and manuscript preparation. YF contributed to data collection, statistical analysis and manuscript preparation. JS and JXZ contributed to data collection and statistical analysis. All authors approved the final version of the manuscript.

\section{Ethics approval and consent to participate}

All patients provided written informed consent and approved the use of their samples in the present study. The study procedures obtained approval from the Ethics Committee at Renmin Hospital.

\section{Patient consent for publication}

Not applicable.

\section{Competing interests}

The authors declare that they have no competing interests.

\section{References}

1. Morera LP, Marchiori GN, Medrano LA and Defagó MD: Stress, dietary patterns and cardiovascular disease: A mini-review. Front Neurosci 13: 1226, 2019.

2. Portegies ML, Koudstaal PJ and Ikram MA: Cerebrovascular disease. Handb Clin Neurol 138: 239-261, 2016.

3. Xiao X, Liu HX, Shen K, Cao W and Li XQ: Canonical transient receptor potential channels and their link with cardio/cerebro-vascular diseases. Biomol Ther (Seoul) 25: 471-481, 2017.

4. Xing F, Dong Y, Tao J, Gao X, Zhou J, Chen S, Ji C, Yao T and $\mathrm{Wu} S$ : Impact of isolated diastolic hypertension on new-onset cardiovascular and cerebro-vascular diseases. Zhonghua Liu Xing Bing Xue Za Zhi 35: 956-960, 2014 (In Chinese).

5. Liu L, Cheng Z and Yang J: miR-23 regulates cell proliferation and apoptosis of vascular smooth muscle cells in coronary heart disease. Pathol Res Pract 214: 1873-1878, 2018.

6. Yoshioka S, Tsukamoto T and Chihara K: Vascular smooth muscle cells in coronary heart disease. Nihon Rinsho 61 (Suppl 4): S80-S85, 2003 (In Japanese).

7. Qiu Z, He Y, Zhang Y, Guo J and Wang L: Characterization of miRNAs and their target genes in $\mathrm{He}-\mathrm{Ne}$ laser pretreated wheat seedlings exposed to drought stress. Ecotoxicol Environ Saf 164 611-617, 2018.

8. Nolan J, Stallings RL and Piskareva O: Assessment of basic biological functions exerted by miRNAs. Methods Mol Biol 1509: 11-16, 2017.
9. Ooi JY, Bernardo BC and McMullen JR: The therapeutic potential of miRNAs regulated in settings of physiological cardiac hypertrophy. Future Med Chem 6: 205-222, 2014.

10. Katz MG, Fargnoli AS, Williams RD, Kendle AP, Steuerwald NM and Bridges CR: MiRNAs as potential molecular targets in heart failure. Future Cardiol 10: 789-800, 2014

11. Wang H, Lu J, Wu S, Yang S, Wang L, Zhou H, Fu Y and Liu J: Effects of electroacupuncture at different acupoints on apoptosis and the expression of miRNAs in myocardial cells in rats model of myocardial ischemia. Zhongguo Zhen Jiu 36: 281-286, 2016 (In Chinese)

12. Gottlieb RA and Pourpirali S: Lost in translation: MiRNAs and mRNAs in ischemic preconditioning and ischemia/reperfusion injury. J Mol Cell Cardiol 95: 70-77, 2016.

13. Maegdefessel L, Spin JM, Raaz U, Eken SM, Toh R, Azuma J, Adam M, Nakagami F, Heymann HM, Chernogubova E, et al: miR-24 limits aortic vascular inflammation and murine abdominal aneurysm development. Nat Commun 5: 5214, 2014.

14. Zheng Y, Li Y, Liu G, Qi X and Cao X: MicroRNA-24 inhibits the proliferation and migration of endothelial cells in patients with atherosclerosis by targeting importin- $\alpha 3$ and regulating inflammatory responses. Exp Ther Med 15: 338-344, 2018.

15. Zhu D, Zhang X, Lin Y, Liang S, Song Z and Dong C: MT1JP inhibits tumorigenesis and enhances cisplatin sensitivity of breast cancer cells through competitively binding to miR-24-3p. Am J Transl Res 11: 245-256, 2019.

16. Wang J, Yin K, Lv X, Yang Q, Shao M, Liu X and Sun H: MicroRNA-24-3p regulates Hodgkin's lymphoma cell proliferation, migration and invasion by targeting DEDD. Oncol Lett 17: 365-371, 2019.

17. Yu G, Jia Z and Dou Z: miR-24-3p regulates bladder cancer cell proliferation, migration, invasion and autophagy by targeting DEDD. Oncol Rep 37: 1123-1131, 2017.

18. Wei W, Peng J and Shen T: Rosuvastatin alleviates ischemia/reperfusion injury in cardiomyocytes by downregulating Hsa-miR-24-3p to target upregulated uncoupling protein 2. Cell Reprogram 21: 99-107, 2019.

19. Chen J, Li HM, Zhang XN, Xiong CM and Ruan JL: Dioscin-induced apoptosis of human $\mathrm{LNCaP}$ prostate carcinoma cells through activation of caspase- 3 and modulation of $\mathrm{Bcl}-2$ protein family. J Huazhong Univ Sci Technolog Med Sci 34: 125-130, 2014.

20. Khawaja NR, Carre M, Kovacic H, Estève MA and Braguer D: Patupilone-induced apoptosis is mediated by mitochondrial reactive oxygen species through Bim relocalization to mitochondria. Mol Pharmacol 74: 1072-1083, 2008.

21. Zhang H, Duan J, Qu Y, Deng T, Liu R, Zhang L, Bai M, Li J, Ning T, Ge S, et al: Onco-miR-24 regulates cell growth and apoptosis by targeting Bcl-2L11 in gastric cancer. Protein Cell 7: 141-151, 2016.

22. Wang Y, Tan M, Li H, Li H and Sun Y: Inactivation of SAG or ROC1 E3 ligase inhibits growth and survival of renal cellcarcinoma cells: Effect of BIM. Transl Oncol 12: 810-818, 2019.

23. Kim JH, Lee DK, Kim J, Choi S, Park W, Ha KS, Kim TH, Choe J, Won MH, Kwon YG and Kim YM: A miRNA-101-3p/Bim axis as a determinant of serum deprivation-induced endothelial cell apoptosis. Cell Death Dis 8: e2808, 2017.

24. Livak KJ and Schmittgen TD: Analysis of relative gene expression data using real-time quantitative PCR and the 2(-Delta Delta C(T)) method. Methods 25: 402-408, 2001.

25. Schwartz M, Bockmann S and Hinz B: Up-regulation of heme oxygenase-1 expression and inhibition of disease-associated features by cannabidiol in vascular smooth muscle cells. Oncotarget 9: 34595-34616, 2018.

26. Torremade N, Bozic M, Panizo S, Barrio-Vazquez S, Fernandez-Martín JL, Encinas M, Goltzman D, Arcidiacono MV, Fernandez E and Valdivielso JM: Vascular calcification induced by chronic kidney disease is mediated by an increase of $1 \alpha$-hydroxylase expression in vascular smooth muscle cells. J Bone Miner Res 31: 1865-1876, 2016.

27. Zhou SS, Jin JP, Wang JQ, Zhang ZG, Freedman JH, Zheng Y and Cai L: miRNAS in cardiovascular diseases: Potential biomarkers, therapeutic targets and challenges. Acta Pharmacol Sin 39: 1073-1084, 2018.

28. Kaneto CM, Nascimento JS, Prado MSJG and Mendonça LSO: Circulating miRNAs as biomarkers in cardiovascular diseases. Eur Rev Med Pharmacol Sci 23: 2234-2243, 2019.

29. Chen WX, Ren LH and Shi RH: Implication of miRNAs for inflammatory bowel disease treatment: Systematic review. World J Gastrointest Pathophysiol 5: 63-70, 2014. 
30. Wang SS, Wu LJ, Li JJ, Xiao HB, He Y and Yan YX: A meta-analysis of dysregulated miRNAs in coronary heart disease. Life Sci 215: 170-181, 2018.

31. Ding L, Wang M, Sun D and Li A: A novel method for identifying potential disease-related miRNAs via a disease-miRNA-target heterogeneous network. Mol Biosyst 13: 2328-2337, 2017.

32. Li FP, Lin DQ and Gao LY: LncRNA TUG1 promotes proliferation of vascular smooth muscle cell and atherosclerosis through regulating miRNA-21/PTEN axis. Eur Rev Med Pharmacol Sci 22: 7439-7447, 2018.

33. Afzal TA, Luong LA, Chen D, Zhang C, Yang F, Chen Q, An W, Wilkes E, Yashiro K, Cutillas PR, et al: NCK associated protein 1 modulated by miRNA-214 determines vascular smooth muscle cell migration, proliferation, and neointima hyperplasia. J Am Heart Assoc 5: e004629, 2016.

34. Wu ZW, Liu YF, Wang S and Li B: Corrigendum miRNA-146a induces vascular smooth muscle cell apoptosis in a rat model of coronary heart disease via NF-kB pathway. Genet Mol Res 14: 18703-18712, 2016.

35. Guo C, Li Y, Zhang R, Zhang Y, Zhao J, Yao J, Sun J, Dong J and Liao L: Protective effect of salidroside against diabetic kidney disease through inhibiting BIM-mediated apoptosis of proximal renal tubular cells in rats. Front Pharmacol 9: 1433, 2018.
36. Kumar A, Ghosh S and Chandna S: Evidence for microRNA-31 dependent Bim-Bax interaction preceding mitochondrial Bax translocation during radiation-induced apoptosis. Sci Rep 5: 15923, 2015.

37. Choi YB and Nicholas J: Bim nuclear translocation and inactivation by viral interferon regulatory factor. PLoS Pathog 6: e1001031, 2010.

38. Pang J, Zhang Z, Zheng T, Yang YJ, Li N, Bai M, Peng Y, Zhang J, Li Q and Zhang B: Association of green tea consumption with risk of coronary heart disease in Chinese population. Int J Cardiol 179: 275-278, 2015.

39. Deng X, Liu Y, Luo M, Wu J, Ma R, Wan Q and Wu J: Circulating miRNA-24 and its target YKL-40 as potential biomarkers in patients with coronary heart disease and type 2 diabetes mellitus. Oncotarget 8: 63038-63046, 2017.

40. Chen J, Benlahrech A, Kelleher P and Patterson S: Increased activity of extrinsic and intrinsic apoptosis pathways in different mononuclear cell types in HIV type 1-infected patients regardless of whether they are depleted in disease. AIDS Res Hum Retroviruses 29: 709-717, 2013.

This work is licensed under a Creative Commons Attribution-NonCommercial-NoDerivatives 4.0 International (CC BY-NC-ND 4.0) License. 